\title{
The challenging diagnosis of dysferlinopathy - a case report
}

\author{
Claudiu Gabriel Socoliuc ${ }^{1,2}$, Alexandra Oprisan ${ }^{1,3}$, Amelia Dobrescu ${ }^{4}$, \\ Emilia Manole ${ }^{2,5}$, Alexandra Eugenia Bastian ${ }^{1,2}$ \\ ${ }_{1}^{1}$ "Carol Davila" University of Medicine and Pharmacy, Bucharest, Romania \\ 2Department of Pathology, Colentina Clinical Hospital, Bucharest, Romania \\ ${ }^{3}$ Department of Neurology, Colentina Clinical Hospital, Bucharest, Romania \\ ${ }^{4}$ Department of Genetics, University of Medicine and Pharmacy, \\ Craiova, Romania \\ ${ }^{5}$ Cellular Biology, Neurosciences and Experimental Myology Laboratory, \\ "Victor Babes" National Institute of Pathology, Bucharest, Romania
}

\begin{abstract}
Objectives. Dysferlinopathies are a group of rare genetic myopathies characterized by muscle weakness and atrophy with four distinct clinical phenotypes: Miyoshi myopathy, limb girdle muscular dystrophy type 2B, distal myopathy with anterior tibial onset and an intermediate proximo-distal phenotype. We report a case of dysferlinopathy and discuss relevant clinical, pathological and genetic data.

Material and methods. We present the case of a 36 years old man with more than ten years history of progressive muscle weakness and atrophies in the distal lower limbs. He had a first clinical evaluation at the age of 24 years and a muscle biopsy was performed but it was inconclusive. On current admission, he presented with clinical suspicion of Charcot-Marie-Tooth disease, but because of increased serum creatine kinase levels and EMG (electromyography) findings of myopathy, another muscle biopsy was recommended. The muscle tissue obtained was analysed by histopathology, immunohistochemistry, and Western blot techniques, and also a genetic confirmation was recommended.

Results. Based on morphological findings, electrodiagnostic study results and clinical context, the diagnosis of primary dysferlinopathy was established, supported by genetic data.

Conclusions. The diagnosis may be challenging in these rare genetic myopathies, due to the high variability of phenotypes, ranging from asymptomatic hyperCKemia (high serum creatine kinase level) to a severe clinical picture with loss of ambulation. Repeated evaluation, increasing access to genetic testing and a multidisciplinary approach made an accurate diagnosis possible in our case.
\end{abstract}

Keywords: dysferlinopathies, Miyoshi myopathy, distal myopathy, limb girdle muscular dystrophy

\section{INTRODUCTION}

The numerous types of muscular dystrophies are caused by various mutations in the genes encoding for the wide spectrum of muscle proteins located in the muscle sarcolemma, basement membrane, sarcomere, myonuclei, extracellular matrix or due to deficits of enzymatic proteins. Dysferlin is one of the proteins located on the subsarcolemmal surface of the skeletal and cardiac muscle fiber and also in the kidney.
Dysferlinopathies represent a group of rare genetic myopathies characterized by muscle weakness and atrophy with four distinct clinical phenotypes: Miyoshi myopathy (MM), a distal myopathy with asymmetric weakness and atrophy especially involving the posterior calf muscles; limb girdle muscular dystrophy type 2B (LGMD2B), affecting the scapular and pelvic girdle muscles; a distal myopathy with anterior tibial onset (DMAT) and an intermediate proximo-distal phenotype (PD) $[1,2]$. 
The initial diagnosis is challenging due to the high variability of clinical phenotypes, that may also change during the disease course, so that the muscle involvement may extend from distal to proximal muscles and inversely, sometimes overlapping. Rare cases may present with a rigid spine syndrome or lax spine due to an early involvement of the paraspinal muscles. Therefore is important to investigate the patients with dysferlin deficiency to better understand both the clinical features and the pathogenic mechanisms that contribute to the disease.

All types of dysferlinopathies are caused by autosomal recessive mutations in the huge dysferlin gene DYSF with 55 exons, located on the chromosome 2p13 [3,4], leading to dysferlin deficiency, a protein with an important role in the efficient repair of muscle sarcolemma. The disease onset usually occurs in the late teens or young adulthood, and progress is slow [2,5], but in some cases a rapid progression to a non-ambulatory state and wheelchair need has been reported. Klinge et al. showed that the age of onset may be more varied than previously appreciated, ranging from early childhood to the fifth to seventh decades of life [5]. There are even reports on rare congenital cases. Currently, there is no specific treatment or cure and disease management aims to improve patients' lives and life expectancy.

Dysferlin is a $237 \mathrm{kDa}$ transmembrane type II protein and encompasses a large, intracytoplasmic $\mathrm{N}$-terminal domain, a transmembrane C-terminal domain and a short extracellular C-terminal domain. The most important functions of dysferlin are the sarcolemma repair, myoblast differentiation, muscle regeneration and T-tubulogenesis, despite the lack of a direct connection to the dystrophin-glycoprotein complex. It is known that muscle fiber is particularly prone to membrane rupture due to repeated contraction-relaxation cycles. Dysferlin-deficient muscles show an accumulation of vesicles near the membrane lesions, suggesting that dysferlin may help the vesicles to fuse with the plasma membrane. Furthermore, in cases of plasma membrane damage, a marked accumulation of dysferlin can be noted at the site, along with other proteins that are believed to be involved in repair, such as annexin [6]. The mechanism of membrane repair is not precisely known, but dysferlin probably bind lipids in a calcium dependent manner [7]. An in vitro study showed that in myotubes dysferlin is located in a cellular compartment that responds to lesion by forming large vesicles containing dysferlin and these vesicles would help repair the membrane [8]. It is assumed that dysferlin interacts with caveolin 3 so that dysferlin is retained in the plasma membrane [9]. Dysferlin interacts with MG53, also involved in membrane repair [10].

Clinical symptoms of dysferlinopathies are varied, from asymptomatic hyperCKemia to the development of a severe clinical picture with functional disability and loss of ambulation [2]. The pattern of muscle involvement is related to the specific phenotype, but the lower limbs are more severely and early affected than the upper limbs in all forms. There is a high intra- and interfamilial variability in both disease progression and pattern of weakness. Surprisingly, in contrast to all other recessive dystrophies, patients had good prior sporting prowess. Also in contrast to other forms of LGMD, which have pseudohypertrophy of the posterior calf muscles, many patients present with early atrophy and weakness of the gastrocnemius and soleus muscles, part of the Miyoshi phenotype. On clinical examination, patients have inability standing on their tiptoes. Another common observation is early loss of the Achilles tendon reflexes, unlike other types of LGMD, where this is the most preserved reflex. DMAT is characterised by early involvement of anterior tibial muscles and rapid progression. In the PD phenotype, both proximal and distal lower limb weakness can be identified. Some patients have transient calf myalgias. Serum creatine kinase (CK) levels are markedly elevated (3,000-10,000 UI/l) especially in the early stages of disease, even in the preclinical period. Cardiac and respiratory involvement are usually not observed, intelligence is normal and life expectancy is unaffected. Electromyography reveals only nonspecific myopathic abnormalities. In the recent years, imaging studies correlated with functional tests identified a selective pattern of muscle involvement in small and even large cohorts of dysferlinopathy patients, and the gastrocnemius medialis and the soleus were the most affected muscles. This pattern is considered as highly characteristic for the disease [11]. 


\section{CASE PRESENTATION}

\section{Clinical data}

We report the case of a 36 years old Caucasian male, who was referred to our hospital for evaluation, with more than ten years old history of progressive muscle weakness and atrophies in his distal lower limbs, gait abnormalities and transient myalgia. He had a first clinical evaluation at the age of 24 years and a muscle biopsy was performed from the left gastrocnemius muscle, but it was inconclusive due to a severe loss of myofibers, almost entirely replaced by fibroadipose tissue, with rare groups of very atrophic muscle fibers, therefore no information about the underlying pathologic process could be provided by the pathologist. The disease slowly progressed over the last years and he gradually lost the strength in the distal lower limbs. No family history of neuromuscular disorders was identified At current admission, he presented with the clinical suspicion of Charcot-Marie-Tooth disease, but because of increased serum creatine kinase levels and EMG (electromyography) findings of myopathy, another muscle biopsy was recommended. He had calf muscle atrophy (Figure 1), distal motor deficit with a severe weakness of foot flexors and extensors, mostly in his left leg, loss of Achilles tendon reflexes, and no sensory impairment. He was unable to stand on toes and heels and had difficulties in walking or climbing stairs. No signs of proximal weakness, scoliosis, rigid spine, facial weakness, myotonia or dysarthria were identified. Electromyography revealed focal myopathy of the calf mus-

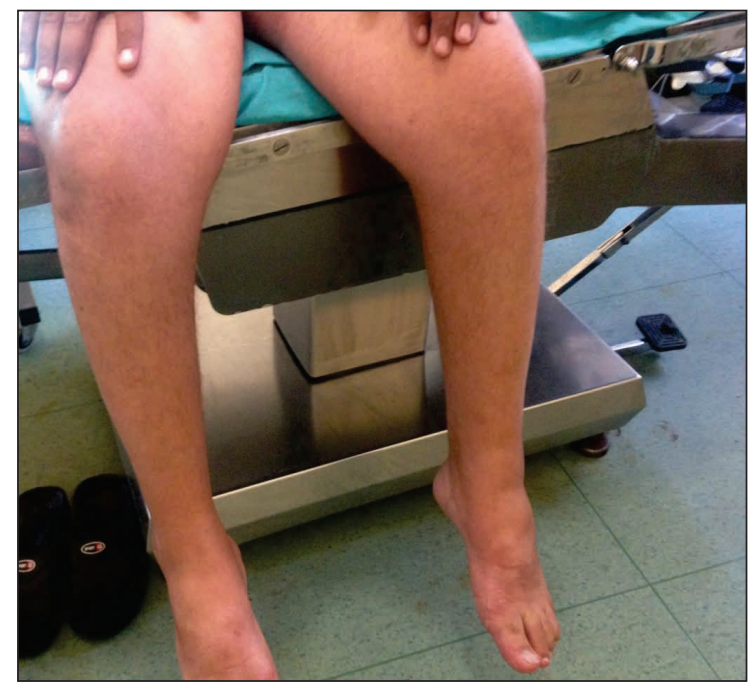

FIGURE 1. An image of the lower limbs showing bilateral calf muscle wasting cles. Serum CK levels were markedly elevated: $8912 \mathrm{U} / 1$ (normal 55-170 U/1), as well as lactate dehydrogenase - $493 \mathrm{U} / 1$ (0-248U/1). A right quadriceps muscle biopsy was required to further evaluate the patient and establish the precise diagnosis.

\section{Muscle biopsy}

A piece of skeletal muscle tissue obtained by open muscle biopsy technique was divided into three fragments: one fragment was immediately frozen in isopentane in liquid nitrogen and cross-sectioned with a cryotome for histological, histochemical and enzyme histochemical preparation: hematoxylin and eosin (HE), van Gieson (VG), modified Gomori Trichrome (MGT), Oil red O, Periodic acid Schiff (PAS), reduced nicotinamide adenine dinucleotide tetrazolium reductase (NADH-TR), succinic and lactic-dehydrogenases (SDH and $\mathrm{LDH}$ ) and adenosine triphosphatase (ATP-ase) at $\mathrm{pH} 9.4,4.63,4.35$; the second portion of tissue was frozen and stored in the ultra-freezer $\left(-80^{\circ} \mathrm{C}\right)$ for Western blotting; a third fragment was formalin-fixed, paraffin embedded and sectioned with a microtome for HE staining. Immunohistochemistry was performed on muscle cryosections for muscular proteins: dystrophin (anti-dys 1, 2, 3 antibodies), utrophin, merosin, dysferlin, alpha-, beta- and gamma-sarcoglycans and major histocompatibility complex class I (MHC I). The control samples were cryosections of muscle biopsy from a patient with peripheral neuropathy. Western blotting analysis was done for dysferlin and calpain-3.

\section{Histopathological exam}

The overall architecture of the skeletal muscle fragments (Figure 2) has been preserved, but the biopsy revealed a dystrophic picture with significant fiber size variability, with atrophy predominance. Rare hypertrophic fibers, necrotic fibers with phagocytosis, regenerated fibers and centralized nuclei were also observed, as well as focal inflammatory infiltrates with lymphocytes in the endo- and perimysium. The internal structure of muscle fibers was unchanged, except for the necrotic ones. Type I and type II fibers were distributed in chessboard normal pattern on ATPases, with atrophy affecting both types of muscle fibers. 


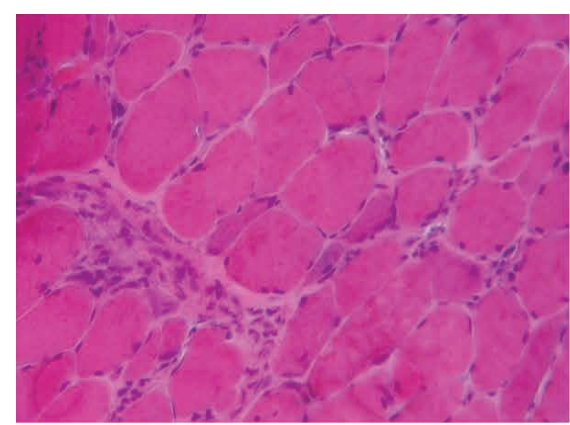

A



D

FIGURE 2. Histopathological features of muscle biopsy. A, B: HE staining: marked variability of muscle fibers with atrophy and mild hypertrophy, without a specific topography; necrotic and regenerated basophilic fibers, internal nuclei are present; focal inflammatory infiltrates. C: PAS staining: no glycogen accumulations were observed.

$D, E, N A D H$ and SDH staining: unchanged internal architecture. F: ATP-ase at pH 9.4: atrophy affects both histoenzymatic fiber types, type I (light) and type II (dark) (muscle transversal cryosections).
Minimal fibroadipose metaplasia has been observed in the paraffin-embedded tissue.

Immunohistochemistry (Figure 3) revealed normal sarcolemmal immunostaining for dystrophin, utrophin, merosin, alpha-, beta- and gamma-sarcoglycans, but dysferlin was absent in most of the muscle fibers, with only a very weak and focal sarcolemmal staining observed in some fibres. A small number of fibers presented a dysferlin weak sarcoplasmic expression. Normal expression of MHC class I was identified on muscle cryosections.

Western blot analysis (Figure 4) showed an extremely weak signal of the dysferlin band (lane 1), highly suggestive for a primary dysferlinopathy, compared with muscular lysates from patients with other forms of myopathy, which presented the normal dysferlin band at $230 \mathrm{kDa}$. Calpain-3 was present in all the samples.

Considering the histopathological picture, the immunohistochemical findings and western blotting analysis, in the clinical context of our patient, a pathological diagnosis of primary dysferlinopathy was established and genetic confirmation was recommended.

\section{Genetic data}

The molecular study showed a homozygous mutation in exon 53 of the DYSF gene: c. $6056 \mathrm{G}>\mathrm{A}$ (p.Arg2019Lys), thus the diagnosis of dysferlinopathy was considered as very likely. The genetic analysis was performed at Cochin University Hospital Paris, Centre for Genetics and Molecular Studies for Neuromuscular Diseases.

\section{DISCUSSION}

Regarding the clinical picture, in dysferlinopathy weakness and atrophy are prominent in the lower limbs. Some authors observations on a cohort of patients with dysferlinopathies, including some initially presenting a limb girdle phenotype, showed that the most severely affected muscle was the gastrocnemius [5]. Fanin et al. showed that Miyoshi myopathy phenotype expresses an evident weakness in distal leg muscles, particularly in gastrocnemius and soleus muscles [12]. Usually the onset is between the ages of 15-30 years and the patients have difficulty standing or walking on their toes [11]. These results are consistent with our observations in the index case, and further- 


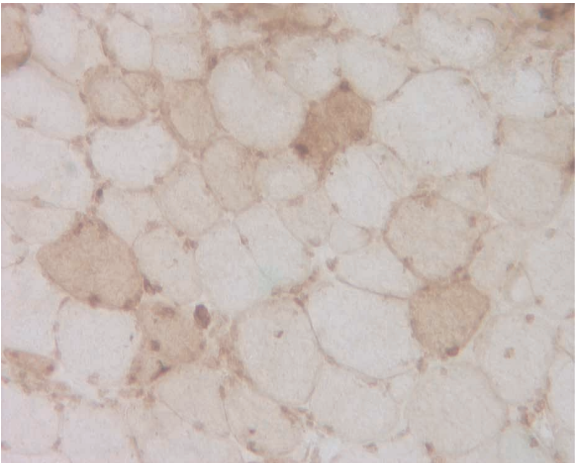

A

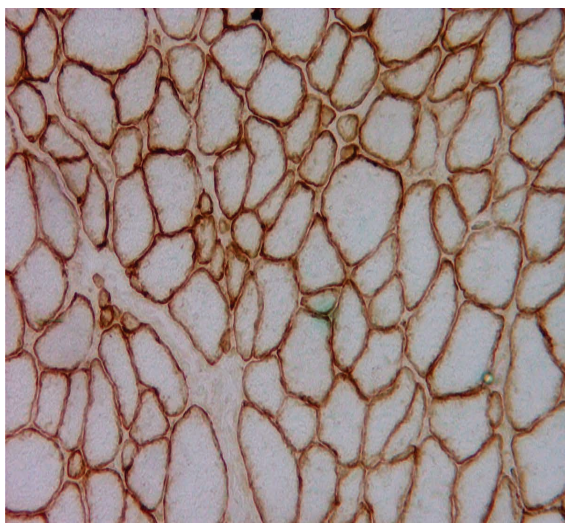

D

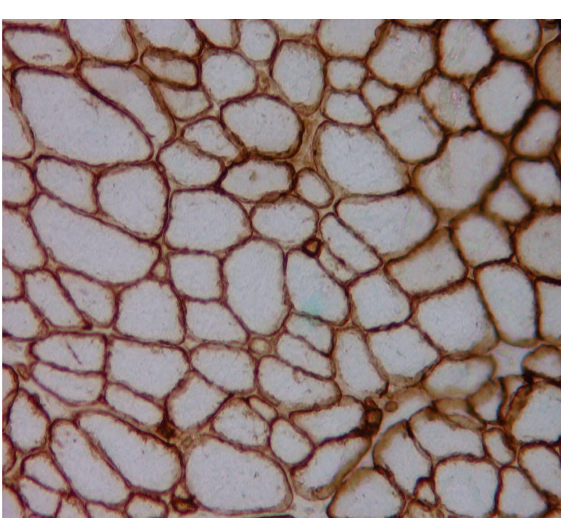

G

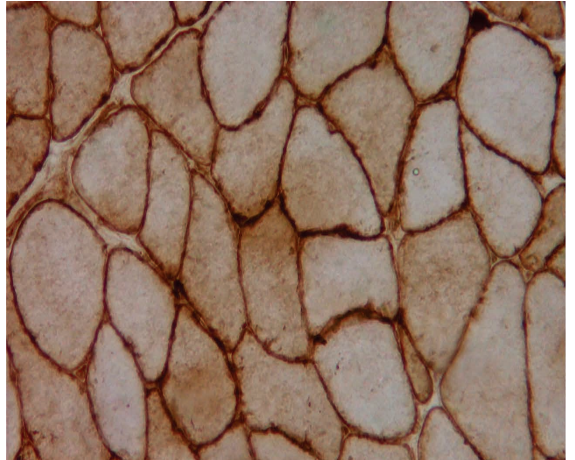

B

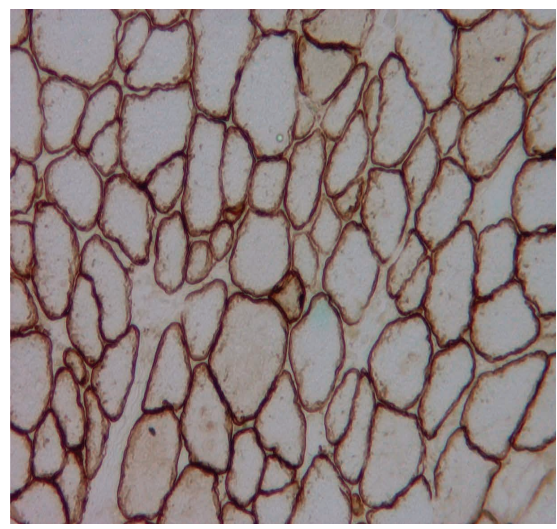

E

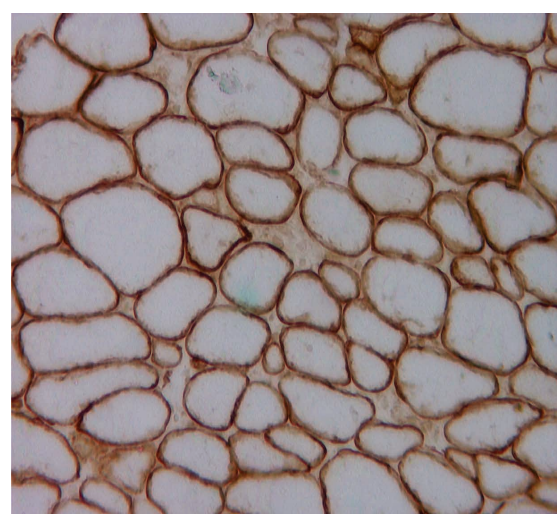

$\mathrm{H}$

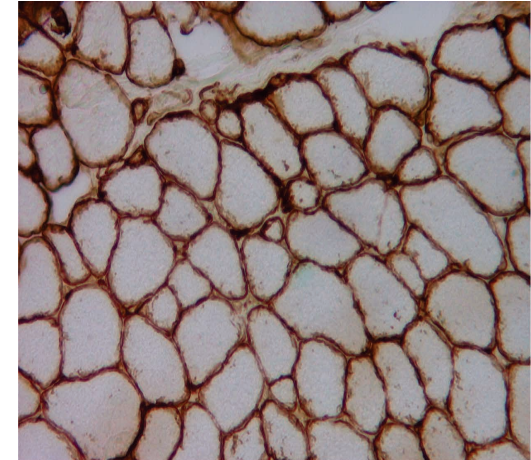

C

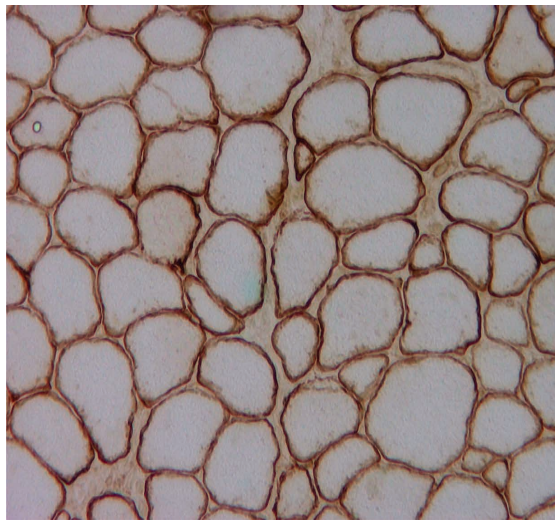

F

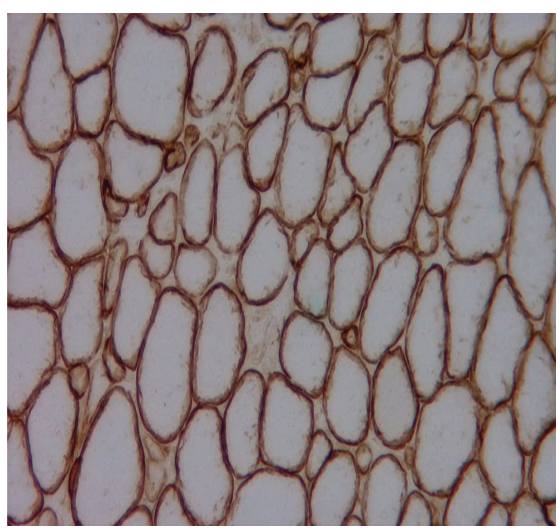

I

FIGURE 3. Immunohistochemistry on muscle biopsy transversal cryosections. $A$, B: Dysferlin absence in the sarcolemma in most fibers (A), compared with control (B). C, D, E, F, G, H, I: all other investigated sarcolemmal proteins (merosin, dystrophin for three different antibodies - D, E, F, alpha-, beta-, gamma-sarcoglycans - G, $H$, I) were normally expressed.

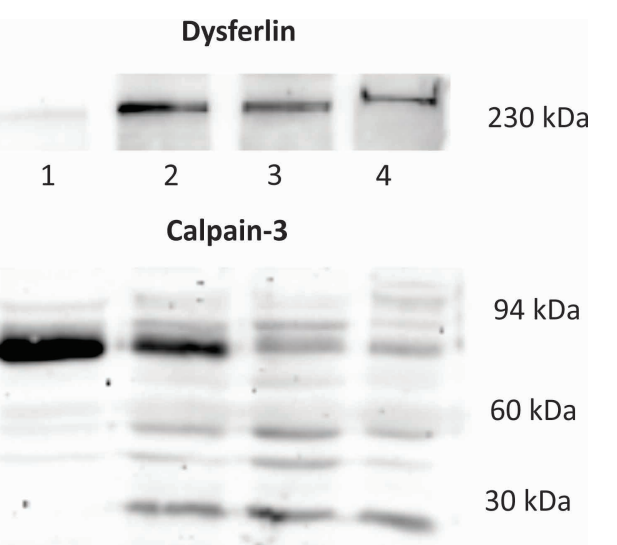

FIGURE 4. Western blot analysis more, considering his first presentation at the age of 24 years, the disease onset in early adulthood is in accordance with the literature data and matches expectations. After an initial enlargement of calf muscles, a marked atrophy follows, sometimes with asymmetrical involvement.

The initial clinical suspicion of CharcotMarie-Tooth, based on the distal involvement, could easily be ruled out even before the biopsy, considering the other clinical aspects and also the markedly elevated CK levels, which directs the diagnosis to a primary muscle disease, not to a neu- 
ropathy. Sparing of the extensor digitorum brevis muscle in dysferlinopathy is a helpful distinctive aspect. Other autosomal recessive limb-girdle muscular dystrophies and other distal myopathies are also part of the differential diagnosis, due to their clinical pictures, but some characteristics of dysferlinopathies are especially useful in the diagnostic algorithm. Of these features, the early weakness and atrophy of the calf muscles is in obvious contrast with the clinical aspects of all others limb-girdle muscular dystrophies in which calf hypertrophy or pseudohypertrophy are prominent features. Again in clear contrast with other LGMDs, where they are typically preserved, early loss of Achille tendon jerks in dysferlinopathies is also very useful to suggest this diagnosis. The absence of significant cardiac and respiratory impairments in these patients should be additional arguments for the positive diagnosis. A history of above average performances in sports before the disease onset is another clue to the diagnosis. On top of all, very high level of serum CK starting early in dysferlinopathies is an additional diagnostic ,red flag".

EMG studies describing myogenic changes are useful, as in our case, to rule out an alternative diagnosis like Charcot-Marie-Tooth, especially in the phenotypes with foot drop like DMAT.

As it was shown by many studies, in dysferlinopathies there is no correlation between phenotype and genotype and the same mutation in DYSF can cause LGMD 2B or MM even in the same family. Mutations found in dysferlinopathies are spread along the entire gene, and no mutational hotspots exist in the dysferlin gene [5]. Mutations in DYSF may cause partial or complete loss of dysferlin. The mutational spectrum is broad, many different mutations were already reported and also high number of sequence variants of unknown pathogenicity.

Concerning the histopathological observations, our patient presented focal inflammatory infiltrates with lymphocytes, known to be present in many muscle biopsies of dysferlinopathy patients, as perivascular infiltrates of mainly $\mathrm{T}$ cells and macrophages around endomysial vessels, but their pathogenic significance is still a matter of debate, and some authors consider them to be secondary changes. MHC class I shows very little or absent sarcolemmal expression in normal looking muscle fibers, and obvious expression in degenerating and regenerating fibers, as has been reported by different authors $[13,14]$. The consistent sarcolemmal upregulation of MHC class I found in inflammatory myopathies is not a prominent feature in dysferlinopathies and is very useful for the differential diagnosis with polymyositis, especially in cases with quite similar pathological pictures. Some retrospective studies showed that up to $25 \%$ of dysferlinopathy patients had been initially misdiagnosed with polymyositis. Previous studies showed that it is no correlation between inflammation status of muscle biopsy and dysferlinopathy phenotype [5]. The presence of inflammatory changes is well described in some of the muscular dystrophies such as Duchenne, but is especially prominent in dysferlinopathies and a differential diagnosis with polymyositis is essential to avoid unnecessary corticosteroid and/or immunosuppresive therapy.

The variability in size of fibers and connective tissue proliferation are not specific for dysferlinopathy, but for many progressive muscular dystrophies. Our case underlines the importance of choosing the proper muscle for biopsy, because in certain cases, a biopsy may be inconclusive as a result of replacement of the muscle by fibroadipose tissue as the disease progresses. Regarding the immunohistochemical data, while in normal muscle fibers dysferlin is localized to the sarcolemma, a reduction in intensity of labelling can be seen in dysferlinopathies. Some internal labelling of fibers also occur, as in our case. These results were correlated with the very low signal in Western blot analysis for dysferlin, practically the protein may be considered absent. It was shown that in most patients series, absence or very important reduction of dysferlin on Western blotting was associated with the abnormal, reduced or absent labelling on immunohistochemistry [11]. Some authors revealed on IHC the presence of dysferlin as scattered granules aggegated in the cytoplasm, even when in the sarcolemma the protein was absent or severely reduced [11]. This is due to a gene mutation that allowed synthesis of a residual quantity of dysferlin, apparent more on IHC labelling than in WB, indicating, as in our case, that the IHC method may be more sensitive than WB in some cases for identification of very low protein levels. On the other hand, immunoblots are important for distinguishing secondary alterations in dysferlin, 
as these can occur when the genes for calpain-3 or caveolin-3 are deffective. Harris et al. reported in 2016 the results of an international multicenter study on 193 patients with dysferlinopathy, in which $68 \%$ had absent and 30\% had reduced dysferlin expression, while normal expression was observed in 3 patients with moderate or severe disease. In this study symptom onset age did not vary according to dysferlin expression levels [15].

\section{CONCLUSIONS}

In recent years significant progress has been made in diagnosing these rare diseases due to in- creasing accessibility to genetic testing. In our case, corroboration of the clinical picture with morphological aspects, electrodiagnostic findings and genetic data, contributed to the final diagnosis of dysferlinopathy, highlighting the importance of a multidisciplinary approach. Establishing an accurate diagnosis in such diseases is essential for the appropriate genetic counselling and patient management, in the perspective of clinical trials. New insights in the pathophysiology of dysferlinopathy will hopefully result in innovative therapies in the years to come.

Conflict of interest: none declared Financial support: none declared

\section{REFERENCES}

1. Aoki M, Takahashi T. Dysferlinopathy. In: Adam MP, Ardinger HH, Pagon RA, et al. (eds.). Seattle (WA): University of Washington, 1993-2021.

2. Nguyen K, Bassez G, Krahn M, et al. Phenotypic Study in 40 Patients With Dysferlin Gene Mutations. Arch Neurol. 2007; 64(8): 1176 .

3. Nguyen K, Bassez G, Bernard R, et al. Dysferlin mutations in LGMD2B, Miyoshi myopathy, and atypical dysferlinopathies. Hum Mutat. 2005;26(2):165.

4. Krahn M, Béroud C, Labelle V, et al. Analysis of the DYSF mutational spectrum in a large cohort of patients. Hum Mutat. 2009;30(2):E345-75.

5. Klinge L, Aboumousa A, Eagle M, et al. New aspects on patients affected by dysferlin deficient muscular dystrophy. $J$ Neurol Neurosurg Psychiatry. 2010;81(9):946-953.

6. Roostalu U, Strähle U. In vivo imaging of molecular interactions at damaged sarcolemma. Dev Cell. 2012;22(3):515-529.

7. Abdullah N, Padmanarayana M, Marty NJ, Johnson CP. Quantitation of the calcium and membrane binding properties of the $\mathrm{C} 2$ domains of dysferlin. Biophys J. 2014;106(2):382-389.

8. McDade JR, Michele DE. Membrane damage-induced vesiclevesicle fusion of dysferlin-containing vesicles in muscle cells requires microtubules and kinesin. Hum Mol Genet. 2014;23(7):1677-1686.

9. Hernández-Deviez DJ, Howes MT, Laval SH, Bushby K, Hancock JF, Parton RG. Caveolin regulates endocytosis of the muscle repair protein, dysferlin. J Biol Chem. 2008;283(10):6476-6488.

10. Cai C, Weisleder N, Ko J-K, et al. Membrane Repair Defects in Muscular Dystrophy Are Linked to Altered Interaction between MG53, Caveolin-3, and Dysferlin. J Biol Chem. 2009; 284(23):15894-15902.

11. Diaz- Manera J, Fernandez-Torron L, Lauger J, et al. Muscle MRI in patients with dysferlinopathy: pattern recognition and implications for clinical trials. J Neurol Neurosurg Psychiatry. 2018;89:1071-1081.

12. Fanin $A$, Angelini $C$. Progress and challenges in diagnosis of dysferlinopathy. Muscle Nerve. 2016;54(5):821-835.

13. Gallardo E, Rojas-Garcia R, de Luna N, et al. Inflammation in dysferlin myopathy: immunohistochemical characterization of 13 patients. Neurology. 2001;57:2136-8.

14. Confalonieri $\mathrm{P}$, Oliva L, Andretta $\mathrm{F}$, et al. Muscle inflammation and $\mathrm{MHC}$ class I up-regulation in muscular dystrophy with lack of dysferlin: an immunopathological study. J Neuroimmunol. 2003; 142:130-6.

15. Harris E, Bladen CL, Mayhew A, et al. The clinical outcome study for dysferlinopathy: An international multicenter study. Neurol Genet. 2016;2(4):e89. 